\title{
Clinically significant interactions between antiretroviral and co-prescribed drugs for HIV- infected children: profiling and comparison of two drug databases
}

\author{
This article was published in the following Dove Press journal: \\ Therapeutics and Clinical Risk Management \\ 13 May 2013 \\ Number of times this article has been viewed
}

\section{Kazeem A Oshikoya' \\ Ibrahim A Oreagba ${ }^{2}$ \\ Olayinka O Ogunleye' \\ Saheed Lawal ${ }^{2}$ \\ Idowu O Senbanjo 3}

'Department of Pharmacology, Lagos State University College of Medicine, Ikeja, Lagos, Nigeria; ${ }^{2}$ Department of Pharmacology, College of Medicine, University of Lagos, Idi Araba, Lagos, Nigeria; ${ }^{3}$ Department of Paediatric and Child Health, Lagos State University College of Medicine, Ikeja, Lagos, Nigeria
Correspondence: Kazeem Adeola Oshikoya

Department of Pharmacology, Lagos State University College of Medicine, PMB 21266, Ikeja, Lagos, Nigeria Tel +234809068 8437

Emailmed_modhospital@yahoo.com
Background: Drug-drug interactions are an important therapeutic challenge among human immunodeficiency virus-infected patients. Early recognition of drug-drug interactions is important, but conflicts do exist among drug compendia on drug interaction information. We aimed to evaluate the consistencies of two drug information resources with regards to the severity rating and categorization of the potential interactions between antiretroviral and co-prescribed drugs.

Methods: We reviewed the case files of human immunodeficiency virus-infected children who were receiving treatment at the human immunodeficiency virus (HIV) clinic of the Lagos University Teaching Hospital, Idi Araba, between January 2005 and December 2010. All of the co-prescribed and antiretroviral drug pairs were screened for potential interactions using the Medscape Drug Interaction Checker and the Monthly Index of Medical Specialties Interaction Checker. Drug-drug interaction (DDI) severity and categorization were rated on a scale of A (no known interaction); B (minor/no action needed); C (moderate/monitor therapy); D (major/therapy modification); and X (contraindicated/avoid combination).

Results: A total of 280 patients were at risk of 596 potential DDIs. The databases showed discrepancies, with Medscape database identifying 504 (84.6\%) and USA MIMS database identifying $302(50.7 \%)$ potential DDIs. Simultaneous identification of DDIs by both databases occurred for only 275 (46.1\%) listed interactions. Both databases have a weak correlation on the severity rating $\left(\mathrm{r}_{\mathrm{s}}=0.45 ; P<0.001\right)$. The most common DDIs identified by the databases were nevirapine and artemisinin-based combination therapy $(170 ; 28.5 \%)$, nevirapine and fluconazole (58; 9.7\%), and zidovudine and fluconazole (55; 9.2\%). There were $272(45.6 \%)$ interaction severity agreements between the databases.

Conclusion: Discrepancies occurred in DDI listings between Medscape and USA MIMS databases. Health care professionals may need to consult more than one DDI information database to ensure safe concomitant prescribing for HIV patients.

Keywords: drug-drug interactions, severity rating, drug interaction checkers, pediatric population, category of interaction, concomitant medication

\section{Introduction}

Drug-drug interactions are an important therapeutic challenge among human immunodeficiency virus-infected patients on antiretroviral drugs. They are often observed in these patients because they frequently receive multiple medications concomitantly with the antiretroviral therapy for treating the numerous infections and systemic consequences of human immunodeficiency virus/acquired immunodeficiency syndrome. ${ }^{1,2}$ 
Highly active antiretroviral (ARV) therapy, defined as the combination of three or more ARV agents taken concurrently to suppress human immunodeficiency virus (HIV) replication, represents the current standard of care of ARV therapy for HIV-infected patients. ${ }^{3}$ This strategy evolved from the recognition that treatment of chronic HIV infection with only one or two ARV drugs typically results in rapid treatment failure and the development of ARV resistance, which may compromise future therapeutic options. ${ }^{3,4}$

An important means of preventing drug-drug interactions in HIV-infected patients is early recognition. This may involve using drug compendia or electronic databases as a source of drug-drug interaction (DDI) information before prescribing. Currently, a number of commercial DDI databases are available; compendia such as MICROMEDEX ${ }^{\circledR 5}$ and Lexicomp ${ }^{\circledR 6}$ are commonly used resources that can provide detailed DDI information including onset, severity, scientific evidence, pharmacologic effects, mechanisms of action, and management. However, these resources may not be available in the developing countries; therefore, alternative electronic databases, such as the Medscape Drug Interaction Checker ${ }^{7}$ and the Monthly Index of Medical Specialties Interaction Checker, ${ }^{8}$ that are available online for free use, may be sought. Although references may be helpful in identifying drug interactions, studies have shown that major conflicts exist among drug compendia on drug interaction information, particularly with regard to specific information such as severity and evidence ratings. ${ }^{9-11}$

The main objective of this study was to identify the clinically significant DDI between ARV and co-prescribed drugs among HIV-infected children in Lagos, Nigeria, and to evaluate the consistencies of the drug information resources with regards to severity ratings and categorization of the DDIs.

\section{Methods}

\section{Study design}

We retrospectively analyzed the clinical records of HIVinfected children who were receiving treatment at the AIDS Prevention Initiative in Nigeria clinic at the Lagos University Teaching Hospital in Nigeria, between January 2005 and December 2010. The AIDS Prevention Initiative in Nigeria clinic at the Lagos State University Teaching Hospital is one of the United States' Presidential Emergency Plan for AIDS Relief-funded centers for the HIV relief program. The clinic is held every Monday through Friday, between 8 am and $4 \mathrm{pm}$.

On average, about 350 returning and new patients (adults and children) are seen each day at the AIDS Prevention
Initiative in Nigeria (APIN) clinic. All HIV-infected children, including those who had progressed to full-blown acquired immunodeficiency syndrome (AIDS) (according to the World Health Organization's criteria), ${ }^{3}$ as well as meeting the other inclusion criteria, were included in this study. These included children younger than 15 years old and those who had been initiated on highly active ARV therapy. Patients must have completely documented demographic information and prescribed medications in the case files. Also, they must have used ARV drugs, at least once, after enrollment.

\section{Data abstraction}

Eligible cases were identified through the main register obtained from the medical record of the APIN clinic. One of the researchers (SL) reviewed each case file, and - using a standard form purposely designed for the study - extracted data on sex, mode of contracting HIV, comorbid diseases and concurrent infections at presentation and follow-up, coprescribed drugs, and the highly active ARV therapy regimen prescribed for each patient.

\section{Prescribed highly active ARV therapy (HAART) regimen}

The national guidelines for HIV treatment in Nigeria ${ }^{12}$ recommended first-line ARV drugs for children such as zidovudine and lamivudine, plus nevirapine or efavirenz; substitution with stavudine or abacavir was allowed for toxicity. Second-line ARV drugs included any of the first-line drugs - didanosine and abacavir or didanosine and zidovudine (AZT) or didanosine (ddI) and efavirenz/ nevirapine - in combination with the protease inhibitors lopinavir/ritonavir or saquinavir/rotinavir.

\section{Identification of potential interactions between co-prescribed and ARV drugs}

The entire co-prescribed and ARV drug pairs were screened for potential interactions using the Medscape Reference Online Drug Interaction Checker ${ }^{7}$ and the Monthly Index of Medical Specialties (MIMS) Interaction Checker. ${ }^{8}$ The potential DDIs not identified by the two databases or those with the severity rated as contraindicated (category X) or unknown (category A) by any of the two databases were searched from a third database, the Liverpool HIV Pharmacology Group website. ${ }^{13}$ This is to ensure that important DDIs were not missed out of the total potential DDIs. 


\section{Classification of potential interactions between co-prescribed and ARV drugs}

The severity and category of interactions were based on the method of Armahizer et al,${ }^{14}$ which utilized MICROMEDEX ${ }^{\circledR 5}$ and Lexicomp ${ }^{\circledR 6}$ method of severity and category classification. The details of the severity rating scale (A to $\mathrm{D}$ and $\mathrm{X}$ ) are presented in Table 1. Interactions relating solely to overlapping toxicities, or between co-prescribed ARV drugs, such as protease inhibitor boosting, or involving topical applications, were excluded. In addition, we excluded from our analysis the potential interactions between lamivudine and cotrimoxazole, due to limited clinical significance suggested by controlled data. ${ }^{2}$ If a given drug interaction was listed more than once with different risk ratings, the most-severe risk rating was used to determine the severity grade. Interaction between ritonavir in the lopinavir/ritonavir combination and the sulfamethoxazole/trimethoprim were excluded from analysis, since these drug combinations are generally used together intentionally and result in a beneficial interaction.

\section{Results}

\section{Demographics of the patients}

A total of 417 patients were enrolled for HAART over the study period, but only $310(74.3 \%)$ met the inclusion criteria. The majority of those excluded had incomplete demographic information or missing details of the ARV or co-prescribed drugs. The case files of all the 310 patients were reviewed in this study. Females $(172 ; 55.5 \%)$ were more affected than males $(138 ; 44.5 \%)$. Their median age was 3 (range 1-15) years. Mother-to-child transmission $(182 ; 58.8 \%)$ and blood transfusion $(10 ; 3.2 \%)$ were the most common routes of transmitting HIV-infection in the study. However, the route of transmission was unknown in $31(10.0 \%)$ cases and not documented in 87 (28.1\%) cases.

\section{Prescribed HAART regimen}

A total of 306 patients (98.7\%) were enrolled on first-line ARV therapy, comprising of zidovudine-lamivudine-(NVP) (279; 91.2\%) and zidovudine-lamivudine-efavirenz (27; 8.8\%). Four (1.3\%) patients were enrolled on a second-line treatment (zidovudine-lamivudine-abacavir (ABC)-lopinavir/ritonavir [LPVr]). The first-line ARV therapies were changed for 66 (21.6\%) patients after an initial enrolment. Nearly all the patients $(64 / 66$; 97\%) who switched their HAART regimen did so after a year of commencing the first-line treatment. Poor adherence and therapeutic failure $(60 / 66 ; 90.9 \%)$, therapeutic failure only $(4 ; 6.1 \%)$, and an adverse drug event $(2 ; 3.0 \%)$ were the reasons for changing the HAART regimen. $\mathrm{ABC}-$ lamivudine (3TC)-LPVr, (20/66; 30.3\%); AZT-3TC-ABCLPV/r (16/66; 24.2\%); AZT-3TC-ABC-ddI-LPV/r, (10/66; 15.2\%); ABC-3TC-NVP, (8/66; 12.1\%); AZT-ABC-LPV/r (6/66; 9.1\%); and AZT-3TC-LPV/r (6/66; 9.1\%) were the types of second-line regimens prescribed.

\section{Co-medications for HIV-infected children on ARV drugs}

A wide range of medications were co-prescribed for the patients while on HAART regimen. The drugs were used to treat comorbid conditions, opportunistic infections, or concurrent infections. Tuberculosis $(35 ; 11.3 \%)$ was the most common opportunistic infection treated in the patients. It was treated with a combination of rifampicin-isoniazidpyrazinamide for an average of six months either before or during ARV treatment. Presumptively diagnosed malaria (208; $67.1 \%)$, pneumonia $(70 ; 22.6 \%)$, and sepsis $(4 ; 1.3 \%)$ were the concurrent infections frequently treated in the patients.

Table I Drug-drug interaction rating scale

\begin{tabular}{llll}
\hline Rating & Category & Action & Explanation \\
\hline X & $\begin{array}{l}\text { Contraindicated } \\
\text { Major }\end{array}$ & $\begin{array}{l}\text { Avoid combination } \\
\text { Consider therapy } \\
\text { modification }\end{array}$ & $\begin{array}{l}\text { The drugs are contraindicated for concurrent use } \\
\text { The interaction may be life threatening and/or require } \\
\text { medical intervention to minimize or prevent serious } \\
\text { adverse events } \\
\text { C }\end{array}$ \\
Moderate & Monitor therapy & $\begin{array}{l}\text { The interaction may result in exacerbation of the patient's } \\
\text { condition and/or require an alteration in therapy }\end{array}$ \\
Minor & No action needed & $\begin{array}{l}\text { The interaction would have limited clinical effects. May } \\
\text { include an increase in the frequency or severity of side } \\
\text { effects but generally would not require a major alteration } \\
\text { in therapy }\end{array}$ \\
Anknown
\end{tabular}

Note: Data from Lexicomp. ${ }^{6}$ MICROMEDEX ${ }^{\otimes 5}$ and Armahizer et al. ${ }^{14}$ 


\section{Identified potential interactions between co-prescribed and ARV drugs}

The first-line regimens, AZT-3TC-NVP (309; 67.1\%) and AZT-3TC-EFV (66; 14.3\%), were frequently associated with clinically significant drug interactions, followed by a secondline regimen, $\mathrm{ABC}-3 \mathrm{TC}-\mathrm{NVP}(30 ; 6.6 \%)$. Other regimens associated with DDIs were ABC-3TC-EFV, $(8 ; 1.7 \%)$; ABC-3TC-AZT-LPV/r, (9; 1.9\%); ABC-3TC-LPV/r, (10; 2.2\%); AZT-3TC-LPV/r, (12; 2.4\%); and AZT-3TC-ddI$\mathrm{LPV} / \mathrm{r},(17 ; 3.7 \%)$.

A total of 310 patients were included in the evaluation, and 596 potential DDIs were identified in $280(67.1 \%)$ patients. Discrepancies between the databases were noted, with the Medscape $^{7}$ electronic database identifying 504 (84.6\%) and the MIMS database ${ }^{8}$ identifying 302 (50.7\%) potential DDIs. Excluding category A interaction (unknown/ no known interaction), simultaneous identification of DDIs by both databases, occurred for only 275 (46.1\%) listed interactions. Table 2 shows the discrepancies in the severity rating and categorization of the interactions identified by the two databases. The Spearman's rank correlation test result suggested a medium correlation between the Medscape database and the MIMS database on the severity rating $\left(\mathrm{r}_{\mathrm{s}}=0.45\right.$; $P<0.001){ }^{7,8}$ The DDIs most commonly identified by the databases were nevirapine (NVP) and artemisinin-based combination therapy (antimalarials), (170; 28.5\%); NVP and fluconazole, $(58 ; 9.7 \%)$; and zidovudine and fluconazole, (55; 9.2\%) (Table 3). Interaction severity agreement differed between the databases, with Medscape and MIMS databases agreeing for 272 (45.6\%) interactions.

An evaluation of contraindicated DDIs was conducted to determine their potential clinical relevance. A total of 189 (31.7\%) contraindicated DDIs were discovered during the evaluation by the Medscape database only and involved NVP and artemisinin-based combination therapy (170; 28.5\%) and efavirenz (EFV) and artemisinin-based combination

Table 2 Severity and category of the interactions between antiretroviral and co-prescribed drugs

\begin{tabular}{llll}
\hline Severity & Category & $\begin{array}{l}\text { Medscape } \\
\text { database n (\%) }\end{array}$ & $\begin{array}{l}\text { MIMS } \\
\text { database n (\%) }\end{array}$ \\
\hline A & Unknown & $96(16.1)$ & $303(50.8)$ \\
B & Minor & $72(12.1)$ & - \\
C & Moderate & $239(40.1)$ & $293(49.2)$ \\
D & Major & - & - \\
X & Contraindicated & $189(31.7)$ & - \\
Total & & $596(100.0 \%)$ & $596(100.0 \%)$ \\
\hline
\end{tabular}

Abbreviations: $n$, number; MIMS, Monthly Index of Medical Specialties. therapy $(19 ; 3.2 \%)$. All the contraindicated DDIs were rated as category A (unknown/no known interaction) by the MIMS database, and as category C (moderate severity/ monitor therapy) by the Liverpool HIV Pharmacology Group website, ${ }^{13}$ another database.

\section{Discussion}

Various databases ${ }^{5-8}$ and compendia ${ }^{15-17}$ are available to evaluate DDIs. The differences in their ratings of the severity and category make it complicated to have a uniform system of evaluating DDIs, especially with respect to severity assessment. The overall agreement between the two databases (Medscape and MIMS) in our study was $45.6 \%$, which was similar to the frequency of agreement for physicians in their assessment of DDIs involving medications that were currently prescribed to patients in the adult cardiac intensive care unit. ${ }^{14}$ In contrast, a lower agreement rate $(39.4 \%)$ has been reported for MICROMEDEX ${ }^{\circledR}$ and Lexicomp ${ }^{\circledR}$ databases in the ratings of the potential DDIs among currently used cardiovascular drugs for adult patients. ${ }^{14}$ Another study ${ }^{18}$ had reported a higher agreement rate $(74.3 \%)$ of severity rating for oral anticancer and nonanticancer drugs between Drug Interaction Facts and MICROMEDEX ${ }^{\circledR}$.

Fulda et $\mathrm{al}^{10}$ have compared the inclusion of drug interactions for five drug classes in five American drug interactions compendia and found that individual interactions were rarely listed in more than one or two of the compendia. Chao and Maibach ${ }^{19}$ reported substantial discrepancies among four American drug compendia for the inclusion of drug interactions on selected at-risk dermatologic drugs. Abarca et $\mathrm{al}^{9}$ assessed the agreement of four American drug interaction compendia for major drug interactions and found a substantial disagreement. In an Australian study, $14 \%-44 \%$ of the drug interactions classified as major in any one compendium were not listed in the other compendia. ${ }^{11}$ The previous comparisons of DDI severity between compendia, databases, or databases and clinicians involved medications frequently used in the adult cardiovascular intensive care units, oral anticancer and nonanticancer drugs, dermatologic drugs, and antihypertensive drugs. ${ }^{9-11,14,18}$ These were contrasting to the DDIs between ARV and co-prescribed drugs evaluated in our study.

Several studies have evaluated DDIs in HIV-infected patients, but - to our knowledge - this is the first study evaluating DDIs in HIV-infected children. ${ }^{1,2,20,21}$ Most of the previous studies relied on a single-drug interaction compendium to identify potential interactions between ARV and co-prescribed drugs. In the few studies where more than one compendium was used, none assessed the consistency 
Table 3 The most common antiretroviral and co-prescribed drugs interactions identified

\begin{tabular}{|c|c|c|c|}
\hline \multirow[t]{2}{*}{ ARV and co-prescribed drug interaction } & \multirow{2}{*}{$\begin{array}{l}\text { Number of DDI } \\
\text { identified } \\
\mathbf{N}=596(\%)\end{array}$} & \multicolumn{2}{|l|}{ Severity } \\
\hline & & Medscape database & MIMS database \\
\hline Nevirapine + artemisinin combination therapy & $170(28.5)$ & $\mathrm{x}$ & A \\
\hline Nevirapine + fluconazole & $58(9.7)$ & $\mathrm{C}$ & $\mathrm{C}$ \\
\hline Zidovudine + fluconazole & $55(9.2)$ & B & $\mathrm{C}$ \\
\hline Zidovudine + rifampicin & $35(5.9)$ & $\mathrm{C}$ & $\mathrm{C}$ \\
\hline Nevirapine + prednisolone & $31(5.2)$ & C & $\mathrm{C}$ \\
\hline Zidovudine + ibuprofen & $27(4.5)$ & A & $\mathrm{C}$ \\
\hline Efavirenz + rifampicin & $27(4.5)$ & $\mathrm{C}$ & $\mathrm{C}$ \\
\hline Zidovudine + clarithromycin & $24(4.0)$ & C & C \\
\hline Nevirapine + clarithromycin & $19(3.2)$ & C & C \\
\hline Lamivudine + frusemide & $19(3.2)$ & A & A \\
\hline Nevirapine + frusemide & $15(2.5)$ & A & A \\
\hline Abacavir + metronidazole & $15(2.5)$ & A & $A$ \\
\hline $\begin{array}{l}\text { Lopinavir/ritonavir + artemisinin-based } \\
\text { combination therapy }\end{array}$ & $15(2.5)$ & C & A \\
\hline Efavirenz + loratadine & $15(2.5)$ & $\mathrm{C}$ & A \\
\hline Efavirenz + artemisinin combination therapy & $14(2.4)$ & $x$ & A \\
\hline Nevirapine + rifampicin & $8(1.3)$ & B & $\mathrm{D}$ \\
\hline Lamivudine + sulfadoxine/pyrimethaine & $8(1.3)$ & A & A \\
\hline Lopinavir/ritonavir + artemisinin/amodiaquine & $7(1.2)$ & $\mathrm{C}$ & A \\
\hline Efavirenz + artemisinin/amodiaquine & $5(0.8)$ & C & $\mathrm{D}$ \\
\hline Efavirenz + clarithromycin & $5(0.8)$ & $\mathrm{C}$ & $\mathrm{C}$ \\
\hline Nevirapine + ketoconazole & $4(0.7)$ & C & C \\
\hline Lopinavir/ritonavir + proguanil & $4(0.7)$ & C & A \\
\hline Lopinavir/ritonavir (solution) + metronidazole & $4(0.7)$ & C & A \\
\hline Lopinavir/ritonavir + loratadine & $4(0.7)$ & A & A \\
\hline Lopinavir/ritonavir + frusemide & $4(0.7)$ & A & A \\
\hline Lopinavir/ritonavir + prednisolone & $4(0.7)$ & C & $\mathrm{C}$ \\
\hline
\end{tabular}

Abbreviations: ARV, antiretroviral; DDI, drug-drug interaction; n, number; MIMS, Monthly Index of Medical Specialties.

of severity ratings using two or more databases for DDI check. ${ }^{20,21}$ Our study identified discrepancies between Medscape and MIMS databases with regard to DDI listings and severity ratings between ARV agents and drugs from other classes (Tables 2 and 3). Although this study was limited to only six ARV drugs (lamivudine, AZT, ABC, NVP, EFV, and LPVr), the results were consistent with those involving oral anticancer and nonanticancer drugs from another study, where a comparative assessment of two interaction compendia (Drug Interaction Facts and MICROMEDEX ${ }^{\circledR}$ ) was done. ${ }^{18}$

The discrepancies in DDI listings between the databases seem to suggest that either MIMS is underreporting or Medscape is overreporting DDIs. One reason could be the difference in time between the data collection and the updating of the database online, as DDIs are continually identified in randomized controlled trials and published in journals. The inability to include the more recent and updated interactions could result in many DDIs being excluded from the MIMS.
Although both databases are available free online, the Medscape database appears to be updated continuously with practice-changing evidence culled daily from journal publications, which results in a shorter lag time between updating and publishing the data. Therefore, Medscape database could have included more recent DDIs involving artemisinin-based combination therapy (antimalarials) and loratadine that were not reported in the MIMS (Table 3). This would suggest a need for health care professionals to consult more than just one DDI information reference source to ensure that it is indeed safe to use certain drugs concomitantly.

Several factors could have contributed to the variations in the ratings of severity between the Medscape and the MIMS databases. Both databases might have obtained information from different sources, unpublished reports by drug companies, reports collated from postmarketing surveillance, and summaries of product characteristics. ${ }^{11}$ Depending on which resource is used, the DDI information provided can be different. For instance, the ratings of DDI severity may be less 
severe in the summary of product characteristics compared with postmarketing surveillance reports, or vice versa. The DDI reports in summaries of product characteristics were likely to be generated from randomized controlled trials and may not be completely representative of the general population. In contrast, a larger and more diverse segment of the general population is involved in postmarketing surveillance studies. ${ }^{22,23}$ Other contributory factors to the discrepancies are the possibility that both databases might have extrapolated the DDI of one co-prescribed drug to other drugs within the same class, based on different assumptions, and the nonstandardized method of classifying DDIs and assessing their clinical relevance. ${ }^{11}$

The inconsistencies in the ratings of DDI severity by two different databases can result in several clinical implications. Health care professionals seeking information about a particular DDI may become confused upon their realization that there is a disagreement in the information provided by different databases. All databases or compendia should have similar DDI information to enable health care professionals to work more efficiently, without the need to search for additional information to clarify the discrepancies observed. This will save health care professionals time that could be spent with patients. Furthermore, the discrepancy in DDI listings can result in potential interactions occurring in patients if the DDIs are not identified in the particular database or compendium used by health care professionals. Such discrepancies may be detrimental, especially in HIV patients, because DDIs involving ARV drug and other drugs may result in increased risk of adverse drug toxicities. Therefore, it is very necessary to solve the problem of inconsistency on DDI listings and severity ratings among drug databases and compendia.

As a means of reducing discrepancies in the listing, categorization and severity rating of DDIs in various databases, Hazlet et $\mathrm{al}^{24}$ have proposed precision analysis of the databases or compendia for sensitivity, specificity, positive, and negative predictive value. Other studies have suggested the use of user-friendly additional criteria to identify and classify DDIs, ${ }^{25}$ and the use of a drug interaction probability scale to assess the DDIs and to assist health care professionals in the assessment of drug interaction-induced adverse outcomes. $^{26}$

The $189(31.7 \%)$ DDIs rated as contraindications (category X) by the Medscape database (NVP + artemisininbased combination therapy and EFV + artemisinin-based combination therapy), and the 45 (7.6\%) DDIs rated as moderately significant interactions (LPVr + artemisinin/ amodiaquine; LPVr + artemisinin-based combination therapy;
LPVr + proguanil; LPVr [solution] + metronidazole; and $\mathrm{EFV}+$ loratadine) that required therapy monitoring, were rated as category A by the MIMS database. However, a third database exclusively used for identifying drug interactions with ARV drugs (the Liverpool HIV Pharmacology Group website) corroborated the severity ratings of the category $\mathrm{X}$ and category C DDIs identified by the Medscape database. These findings therefore underscore the importance of using a third database where one of the two databases identifies a DDI as category A or X.

There are some limitations that characterized our study. Only two free drug online electronic databases were used to compile the DDI profiles. Although the results could have been different if other electronic software for DDI checking or compendia had been used, unfortunately, the widely used MICROMEDEX ${ }^{\circledR}$ and Lexicomp ${ }^{\circledR}$ software programs and the compendia were unavailable to us when the study was conducted. Relative to the wide range of ARV drugs available for HIV treatment, only a few ARV drugs were evaluated for DDIs in this study. This was as a result of the national treatment guideline that recommended the use of only the following ARV drugs: AZT; lamivudine; NVP; EFV; stavudine; ABC; ddI; LPVr; or saquinavir/rotinavir for children. Therefore, this study might not have captured the important potential DDIs that are associated with other ARV drugs.

\section{Conclusion}

Discrepancies occurred in DDI listings between the Medscape and the MIMS databases. Health care professionals may need to consult more than one DDI information database to ensure safe concomitant prescribing for HIV patients. Further studies should be conducted to create a standard evaluation tool or selection criteria to standardize the definitions and classifications of DDIs among databases commonly used to identify DDIs. As more ARV drugs are introduced into patients' therapy, clinically significant DDIs should be prevented or identified for the benefit of better care and medication safety for HIV patients.

\section{Disclosure}

The authors report no conflicts of interest in this work.

\section{References}

1. Evans-Jones JG, Cottle LE, Back DJ. Recognition of risk for clinically significant drug interactions among HIV-infected patients receiving antiretroviral therapy. Clin Infect Dis. 2010;50(10):1419-1421.

2. Kigen G, Kimaiyo S, Nyandiko W, et al. Prevalence of potential drugdrug interactions involving antiretroviral drugs in a large Kenyan cohort. PLOS ONE. 2011;6(2):e16800. 
3. World Health Organization. Antiretroviral Therapy of HIV Infection in Infants and Children: Towards Universal Access. Geneva: World Health Organization; 2006. Available from: http://www.who.int/hiv/ pub/guidelines/paediatric020907.pdf. Accessed October 22, 2011.

4. Jordan R, Gold L, Cummins C, Hyde C. Systematic review of metaanalysis of evidence for increasing numbers of drugs in antiretroviral combination therapy. BMJ. 2002;324(7340):757

5. MICROMEDEX ${ }^{\circledR}$ Gateway [database on the Internet]. Greenwood Village, CO, USA: Thomson Reuters; 2013. Available from: http:// www.thomsonhc.com/home. Accessed February 12, 2013.

6. Lexicomp ${ }^{\circledR}$ [database on the Internet]. Hudson, OH, USA: Lexicomp, Inc; 2013. Available from: http://webstore.lexi.com/Information/ Product-Information/Lexi-Drugs-and-Lexi-Interact-Details. Accessed February 12, 2013.

7. Medscape Reference Online Drug Interaction Checker [database on the Internet]. New York, NY, USA: WebMD LLC; 2013. Available from: http://reference.medscape.com/drug-interactionchecker. Accessed February 13, 2013.

8. Monthly Index of Medical Specialties (MIMS) Interaction Checker [database on the Internet]. London, United Kingdom: UBM plc; 2013. Available from: http://www.mims.com/USA/interaction/ AdvancedSearch/. Accessed February 13, 2013.

9. Abarca J, Malone DC, Armstrong EP, et al. Concordance of severity ratings provided in four drug interaction compendia. JAm Pharm Assoc (2003). 2004;44(2):136-141.

10. Fulda TR, Valuck RJ, Zanden JV, Parker S, Byrns PJ. Disagreement among drug compendia on inclusion and ratings of drug-drug interactions. Curr Ther Res Clin Exp. 2000;61(8):540-548.

11. Vitry AI. Comparative assessment of four drug interaction compendia. Br J Clin Pharmacol. 2007;63(6):709-714.

12. Federal Ministry of Health, Nigeria. National Guidelines for Paediatric HIV and AIDS Treatment and Care. Abuja: Federal Ministry of Health; 2007. Available from: http://www.who.int/hiv/amds/ Nigeria_paediatric_2007.pdf. Accessed November 30, 2012.

13. Liverpool HIV Pharmacology Group [database on the Internet]. Cheshire, United Kingdom: The University of Liverpool and eMedFusion; 2010. Available from: http://www.hiv-druginteractions.org. Accessed December 1, 2012.
14. Armahizer MJ, Kane-Gill SL, Smithburger PL, Anthes AM, Seybert AL Comparing drug-drug interaction severity for clinician opinion to propriety databases. ISRN Critical Care. 2013:Article ID 347346.

15. Tatro DS. Drug interaction facts. In: The Authority on Drug Interactions. St Louis, MO: Wolters Kluwer Health Facts and Comparisons; 2008.

16. Baxter K, Preston C. Stockley's Drug Interactions, 10th ed. London: The Pharmaceutical Press; 2013.

17. Joint Formulary Committee. British National Formulary, 61st ed. London: British Medical Association and Royal Pharmaceutical Society of Great Britain; 2011.

18. Wong CM, Ko Y, Chan A. Clinically significant drug-drug interactions between oral anticancer agents and non-anticancer agents: profiling and comparison of two drug compendia. Ann Pharmacother. 2008;42(12): 1737-1748.

19. Chao SD, Maibach HI. Lack of drug interaction conformity in commonly used drug compendia for selected at-risk dermatologic drugs. Am J Clin Dermatol. 2005;6(2):105-111.

20. Yiu P, Nguyen NN, Holodniy M. Clinically significant drug interactions in younger and older human immunodeficiency virus-positive patients receiving antiretroviral therapy. Pharmacother. 2011;31(5): 480-489.

21. Miller CD, El-Kholi R, Faragon JJ, Lodise TP. Prevalence and risk factors for clinically significant drug interactions with antiretroviral therapy. Pharmacotherapy. 2007;27(10):1379-1386.

22. Martin K, Begaud B, Latry P, Miremont-Salame G, Fourrier A, Moore N. Differences between clinical trials and postmarketing use. Br J Clin Pharmacol. 2004;57(1):86-92.

23. Pascual J, Diener HC, Massiou H. Value of postmarketing surveillance studies in achieving a complete picture of antimigraine agents: using almotriptan as an example. J Headache Pain. 2006;7(1):27-33.

24. Hazlet TK, Lee TA, Hansten PD, Horn JR. Performance of community pharmacy drug interaction software. J Am Pharm Assoc (Wash). 2001;41(2):200-204.

25. Hansten PD, Horn JR, Hazlet TK. ORCA: OpeRational ClassificAtion of drug interactions. J Am Pharm Assoc (Wash). 2001;41(2):161-165.

26. Horn JR, Hansten PD, Chan LN. Proposal for a new tool to evaluate drug interaction cases. Ann Pharmacother. 2007;41(4):674-680.
Therapeutics and Clinical Risk Management

\section{Publish your work in this journal}

Therapeutics and Clinical Risk Management is an international, peerreviewed journal of clinical therapeutics and risk management, focusing on concise rapid reporting of clinical studies in all therapeutic areas, outcomes, safety, and programs for the effective, safe, and sustained use of medicines. This journal is indexed on PubMed Central, CAS,

\section{Dovepress}

EMBase, Scopus and the Elsevier Bibliographic databases. The manuscript management system is completely online and includes a very quick and fair peer-review system, which is all easy to use. Visit http://www.dovepress.com/testimonials.php to read real quotes from published authors. 